\title{
IDENTITIES INVOLVING THE COEFFICIENTS OF A CLASS OF DIRICHLET SERIES. VII
}

\author{
BY \\ BRUCE C. BERNDT (1)
}

\begin{abstract}
Let $a(n)$ be an arithmetical function, and consider the Riesz sum $A_{\rho}(x)=\Sigma_{n \leqslant x} a(n)(x-n)^{\rho}$. For $a(n)$ belonging to a certain class of arithmetical functions, $A_{\rho}(x)$ can be expressed in terms of an infinite series of Bessel functions. K. Chandrasekharan and R. Narasimhan have established this identity for the widest known range of $\rho$. Their proof depends upon equi-convergence theory of trigonometric series. An alternate proof is given here which uses only the classical theory of Bessel functions.
\end{abstract}

1. Introduction. Let $a(n)$ denote an arithmetical function. In the study of the average order of arithmetical functions, and in other related problems, an expression for

$$
\sum_{n \leq x} a(n)(x-n)^{\rho}
$$

in terms of an infinite series of analytic functions is often very useful, where $\rho$ assumes certain real values. See, for example, several papers of E. Landau [28], many results of G. H. Hardy [14, part 2], and a paper of K. Chandrasekharan and R. Narasimhan [6]. The case $\rho=0$ is of particular importance. It is of interest to establish the identity for (1.1) for the widest range of $\rho$ possible. The most significant result in this direction is a theorem of Chandrasekharan and Narasimhan [5, Theorem III]. In this result, $a(n)$ is generated by a Dirichlet series satisfying a functional equation involving a simple gamma factor, $\Gamma(s)$. The representation of (1.1) is then given in terms of an infinite series of ordinary Bessel functions. The method was also used by Chandrasekharan and Narasimhan $[6, \S 7]$ and the author [3] to establish identities for (1.1) for arithmetical functions generated by Dirichlet series satisfying certain other functional equations involving the gamma function. The method of Chandrasekharan and

Received by the editors December 18, 1973.

AMS (MOS) subject classifications (1970). Primary 10H10; Secondary 10H25, $10 \mathrm{~J} 25$.

Key words and phrases. Dirichlet series, arithmetical function, functional equation involving gamma factors, Bessel function identity, Hardy-Landau circle method, average order of arithmetical functions, Riesz sum.

(1) Supported in part by NSF grant GP $36418 \times 1$. 
Narasimhan is quite deep and depends upon results of A. Zygmund on the equiconvergence of trigonometric integrals.

The purpose of this paper is to give a different proof of the aforementioned principal theorem of Chandrasekharan and Narasimhan [5, Theorem III]. In contrast to their proof, ours uses only well-known, classical results in the theory of Bessel functions. In fact, the method is merely an extension and elaboration of a method of Hardy and Landau ([15], [28, pp. 95-109] , [14, pp. 329-343], $\left[22\right.$, pp. 189, 225-232]) used in the case when $\rho=0$ and $a(n)=r_{2}(n)$, the number of representations of $n$ as the sum of two squares. The method was also used by Hardy ([13] , [14, pp. 369-375]) to establish an identity for (1.1) when $\rho=0$ and $a(n)=\tau(n)$, Ramanujan's arithmetical function. Lastly, the method was used by the author in his thesis [2] to establish identities for (1.1) when $\rho=0$ and the Dirichlet series is attached to a cusp form. We remark that our hypotheses are slightly different from those of Chandrasekharan and Narasimhan [5]. However, all of the known, particular examples of arithmetical functions generated by Dirichlet series satisfying the functional equation (2.1) below are covered by both theorems.

2. Definition and preliminary results. We first indicate those arithmetical functions to be studied.

Definition 1. Let $\left\{\lambda_{n}\right\}$ and $\left\{\mu_{n}\right\}, 1 \leqslant n<\infty$, be two sequences of positive numbers strictly increasing to $\infty$, and let $\{a(n)\}$ and $\{b(n)\}, 1 \leqslant$ $n<\infty$, be two sequences of complex numbers not identically zero. Assume that the Dirichlet series

$$
\phi(s)=\sum_{n=1}^{\infty} a(n) \lambda_{n}^{-s} \text { and } \psi(s)=\sum_{n=1}^{\infty} b(n) \mu_{n}^{-s}
$$

each converge in some half-plane of the complex s-plane, and let their abscissae of absolute convergence be denoted by $\sigma_{a}$ and $\sigma_{a}^{*}$, respectively. If $r$ is real, we say that $\phi$ and $\psi$ satisfy the functional equation

$$
\Gamma(s) \phi(s)=\Gamma(r-s) \psi(r-s)
$$

if there exists in the s-plane a domain $D$ which is the exterior of a compact set $S$, such that in $D$ a holomorphic function $\chi$ exists with the properties:

$$
\begin{aligned}
\chi(s) & =\Gamma(s) \phi(s) & & \left(\sigma>\sigma_{a}\right) \\
& =\Gamma(r-s) \psi(r-s) & & \left(\sigma<r-\sigma_{a}^{*}\right),
\end{aligned}
$$

$$
\lim _{|t| \rightarrow \infty} \chi(\sigma+i t)=0 \text {, }
$$

uniformly in every strip $-\infty<\sigma_{1} \leqslant \sigma \leqslant \sigma_{2}<\infty$. Here, and in the sequel, $\sigma=\operatorname{Re}(s)$ and $t=\operatorname{Im}(s)$. 
We now list some results on Bessel functions to be used in the sequel. The ordinary Bessel function of order $\nu, J_{\nu}(x)$, may be defined by $[29$, p. 40]

$$
J_{\nu}(x)=\sum_{n=0}^{\infty} \frac{(-1)^{n}(x / 2)^{\nu+2 n}}{n ! \Gamma(\nu+n+1)} \quad(|x|<\infty) .
$$

We shall frequently use the property $[29$, p. 45]

$$
\frac{d}{d x}\left\{x^{-\nu / 2} J_{\nu}\left(c x^{1 / 2}\right)\right\}=-1 / 2 c x^{-(\nu+1) / 2} J_{\nu+1}\left(c x^{1 / 2}\right) \text {. }
$$

As $x$ tends to $+\infty$, we have the asymptotic formula [29, p. 199]

$$
J_{\nu}(x)=(2 / \pi x)^{1 / 2} \cos (x-\pi \nu / 2-\pi / 4)+O\left(x^{-3 / 2}\right) .
$$

Lastly, we need two results on integrals of Bessel functions. Firstly, for $0<$ $2 \sigma<\nu+3 / 2[9$, p. 22]

$$
\int_{0}^{\infty} x^{2 s-\nu-1} J_{\nu}(x) d x=\frac{2^{2 s-\nu-1} \Gamma(s)}{\Gamma(\nu+1-s)} .
$$

(In [9], the conditions on $\sigma$ are given by $0<2 \sigma<\nu+1$. However, using (2.4), one can readily show by analytic continuation that the validity of (2.5) can be extended to $0<2 \sigma<\nu+3 / 2$.) Secondly, for $a>0$ and $\nu>\mu>$ $-1[9$, p. 48], [29, p. 406],

$$
\begin{array}{rlr}
\int_{0}^{\infty} x^{\mu-\nu+1} J_{\mu}(a x) J_{\nu}(x) d x & \\
& =\frac{2^{\mu-\nu+1} a^{\mu}\left(1-a^{2}\right)^{\nu-\mu-1}}{\Gamma(\nu-\mu)}, & a<1, \\
& =0, & a>1, \\
& =0, & \nu>\mu+1, a=1, \\
& =1 / 2, & \nu=\mu+1, a=1 .
\end{array}
$$

3. Main theorem. For real $\rho$, let

$$
A_{\rho}(x)=\frac{1}{\Gamma(\rho+1)} \sum_{\lambda_{n}<x}^{\prime} a(n)\left(x-\lambda_{n}\right)^{\rho},
$$

where the prime on the summation sign indicates that if $\rho=0$ and $x=\lambda_{m}$, $a(m)$ is to be multiplied by $1 / 2$. We define $A_{\rho}^{*}(x)$ by replacing $a(n)$ by $b(n)$ and $\lambda_{n}$ by $\mu_{n}$ above. Next, define

$$
Q_{\rho}(x)=\frac{1}{2 \pi i} \int_{C} \frac{\Gamma(s) \phi(s)}{\Gamma(s+\rho+1)} x^{s+\rho} d s,
$$

where $C$ is a cycle with $S$ on its interior. Similarly, put

$$
Q_{\rho}^{*}(x)=\frac{1}{2 \pi i} \int_{C^{*}} \frac{\Gamma(s) \psi(s)}{\Gamma(s+\rho+1)} x^{s+\rho} d s,
$$


where $C^{*}$ is a cycle containing all singularities of $\Gamma(s) \psi(s)$ on its interior. Furthermore, let

$$
P_{\rho}(x)=A_{\rho}(x)-Q_{\rho}(x) \text { and } P_{\rho}^{*}(x)=A_{\rho}^{*}(x)-Q_{\rho}^{*}(x) .
$$

Lastly, for $x, y>0, \rho$ real, and an arbitrary nonnegative integer $\nu$, let

$$
F_{\nu, \rho}(x, y)=P_{\nu}^{*}(y)(x / y)^{(r+\rho+\nu) / 2} J_{r+\rho+\nu}\left(2\{x y\}^{1 / 2}\right) .
$$

In our first version of the main theorem, we shall assume that the singularities of $\psi(s)$ lie in the right half-plane $\sigma>0$. All known examples satisfy this hypothesis. In the second version of the main theorem, we show that this requirement on $\psi$ may be deleted in most cases.

THEOREM 1. Let $\phi$ and $\psi$ satisfy Definition 1. Assume that the singularities of $\psi(s)$ lie in $\sigma>0$. Suppose that for $2 \sigma_{a}^{*}-r-3 / 2<q \leqslant 2 \sigma_{a}^{*}-$ $r-1 / 2$,

$$
\sum_{0<j<2 \sigma_{a}-r-1 / 2} F_{j, q}(x, N) \rightarrow 0
$$

uniformly on each finite interval $0<x_{1} \leqslant x \leqslant x_{2}$ as $N$ tends to $\infty$. Then for $\rho>2 \sigma_{a}^{*}-r-3 / 2$,

$$
P_{\rho}(x)=\sum_{n=1}^{\infty} b(n)\left(x / \mu_{n}\right)^{(r+\rho) / 2} J_{r+\rho}\left(2\left\{\mu_{n} x\right\}^{1 / 2}\right) .
$$

If $\rho>0$, the infinite series on the right side of (3.3) converges uniformly on $\left[x_{1}, x_{2}\right]$. If $\rho \leqslant 0$, the series converges uniformly on $\left[x_{1}, x_{2}\right]$, provided that $\left[x_{1}, x_{2}\right]$ contains no member of $\left\{\lambda_{n}\right\}$. If $\rho=0$, the series converges boundedly on $\left[x_{1}, x_{2}\right]$.

Before proving Theorem 1, we shall discuss the hypothesis (3.2). From (2.4) and (3.1), we see that (3.2) is equivalent to showing that for each integer $j, 0 \leqslant j \leqslant 2 \sigma_{a}-r-1 / 2$,

$$
P_{j}^{*}(N)=o\left(N^{(r+q+j) / 2+1 / 4}\right)
$$

as $N$ tends to $\infty$, uniformly on $\left[x_{1}, x_{2}\right]$, where $2 \sigma_{a}^{*}-r-3 / 2<q \leqslant 2 \sigma_{a}^{*}$ $-r-1 / 2$. In most applications, (3.4) is quite easy to show. One does not normally need sophisticated estimates, as the examples at the end of this paper will show. In the case $j=0$, general theorems have been proven by Landau ([21], [28, pp. 30-64]) and by Chandrasekharan and Narasimhan [6, Theorem 4.1] that give $O$-estimates for $P_{0}^{*}(N)$ when the singularities of $\psi$ are at most 
poles. For our purposes, it is unfortunate that these results were not proven for $j>0$ as well, for Landau's method of finite differences used in [21] and [6] can be easily applied in the more general situation.

Now, for $j>2 \sigma_{a}-r-1 / 2$, we see from (3.3) (with $P_{j}$ replaced by $P_{j}^{*}$ ) and (2.4) that

$$
P_{j}^{*}(N)=O\left(N^{(r+j) / 2-1 / 4}\right),
$$

uniformly on $\left[x_{1}, x_{2}\right]$. Using the aforementioned results of either Landau or Chandrasekharan and Narasimhan along with (3.5) and a convexity theorem, $P_{j}^{*}(N)$ can be estimated for those $j$ (if any) such that $1 \leqslant j \leqslant 2 \sigma_{a}-r-1 / 2$. The convexity theorem that one needs is a slight generalization of a convexity theorem of M. Riesz [4, p. 13].

Observe that the corresponding theorem of Chandrasekharan and Narasimhan [5, Theorem III] does not have hypothesis (3.2) but another condition [5, equation (48)] instead. The different hypotheses are probably related.

Proof of TheOREM 1. First, a proof of (3.3) for $\rho>2 \sigma_{a}^{*}-r-1 / 2$ is not difficult [5, Lemma 5]. (The method used in [5], as noted there, is apparently due to E. Hecke [16]. We remark that the method was subsequently improved and generalized by A. L. Dixon and W. L. Ferrar [7] and T. M. Apostol [1].) Hence, we shall assume for the remainder of the proof that $\rho=q$, where $2 \sigma_{a}^{*}-r-3 / 2<q \leqslant 2 \sigma_{a}^{*}-r-1 / 2$.

Define for $x, N>0$ and each nonnegative integer $j$,

$$
I_{j}(x, N)=\int_{0}^{N} P_{j}^{*}(u)(x / u)^{(r+q+j+1) / 2} J_{r+q+j+1}\left(2\{u x\}^{1 / 2}\right) d u .
$$

From the definition of $P_{j}^{*}(u)$, it is not difficult to see that $P_{j}^{*}(u)$ is continuous at $u=0$ Thus, from (2.2), $I_{j}$ is well defined. From the definition of $P_{j}^{*}(u)$, it is also clear that $\int P_{j}^{*}(u) d u=P_{j+1}^{*}(u), j \geqslant 0$. Hence, integrating by parts with the aid of (2.3), we find that

$$
I_{0}(x, N)=F_{1, q}(x, N)-F_{1, q}(x, 0)+I_{1}(x, N) .
$$

Choose a positive integer $m$ such that

(A1) $m>2 \sigma_{a}-r-1 / 2$,

(A2) $m+r>-1$,

(A3) all singularities of $\Gamma(s) \psi(s)$ lie in the half-plane $\sigma \geqslant-m$.

After $m$ such integrations by parts, we get

$$
I_{0}(x, N)=\sum_{j=1}^{m}\left\{F_{j, q}(x, N)-F_{j, q}(x, 0)\right\}+I_{m}(x, N) .
$$

We have assumed that (3.3) is valid for $\rho>2 \sigma_{a}^{*}-r-1 / 2$. Hence, by (A1) we have 


$$
P_{m}^{*}(u)=\sum_{n=1}^{\infty} a(n)\left(u / \lambda_{n}\right)^{(r+m) / 2} J_{r+m}\left(2\left\{\lambda_{n} u\right\}^{1 / 2}\right) .
$$

Substituting (3.7) into $I_{m}(x, N)$ and then inverting the order of summation and integration by absolute convergence, we arrive at

$$
\begin{aligned}
I_{m}(x, N)= & x^{(r+q+m+1) / 2} \sum_{n=1}^{\infty} a(n) \lambda_{n}^{-(r+m) / 2} \\
& \times \int_{0}^{N} u^{-(q+1) / 2} J_{r+m}\left(2\left\{\lambda_{n} u\right\}^{1 / 2}\right) J_{r+q+m+1}\left(2\{u x\}^{1 / 2}\right) d u .
\end{aligned}
$$

Observe that the integrals above exist by (2.2) and (A2). Letting $v=2(u x)^{1 / 2}$, we get

$$
\begin{aligned}
I_{m}(x, N)= & (2 x)^{q} \sum_{n=1}^{\infty} a(n)\left(x / \lambda_{n}\right)^{(r+m) / 2} \\
& \times\left\{\int_{0}^{\infty}-\int_{2(N x)^{1 / 2}}^{\infty}\right\} v^{-q} J_{r+m}\left(\left(\lambda_{n} / x\right\}^{1 / 2} v\right) J_{r+q+m+1}(v) d v .
\end{aligned}
$$

We must show that the integrals above exist. Suppose that $\left[x_{1}, x_{2}\right]$ is an interval containing at least one element $\lambda_{j}$ with $a(j) \neq 0$. For $\rho>2 \sigma_{a}^{*}-$ $r-1 / 2$, the right side of (3.3) converges absolutely and uniformly on $\left[x_{1}, x_{2}\right]$. Thus, the left side of (3.3) is continuous on $\left[x_{1}, x_{2}\right]$. Since $\left[x_{1}, x_{2}\right]$ contains $\lambda_{j}$ with $a(j) \neq 0$, then necessarily $\rho>0$, which implies that $2 \sigma_{a}^{*}-r$ $-1 / 2 \geqslant 0$. Thus, in all cases $q>-1$. Hence, using (2.4), we see that the infinite integrals on the right side of (3.8) converge.

We now apply (2.6) with $a=\left\{\lambda_{n} / x\right\}^{1 / 2}, \mu=r+m$, and $\nu=r+q+$ $m+1$. By (A2) and the fact that $q>-1$, we see that $\nu>\mu>-1$. Hence, from (3.8),

$$
I_{m}(x, N)=\frac{1}{\Gamma(q+1)} \sum_{\lambda_{n}<x}^{\prime} a(n)\left(x-\lambda_{n}\right)^{q}-K(x, N),
$$

where

$$
\begin{aligned}
K(x, N)= & (2 x)^{q} \sum_{n=1}^{\infty} a(n)\left(x / \lambda_{n}\right)^{(r+m) / 2} \\
& \times \int_{2(N x)^{1 / 2}}^{\infty} v^{-q} J_{r+m}\left(\left\{\lambda_{n} / x\right\}^{1 / 2} v\right) J_{r+q+m+1}(v) d v .
\end{aligned}
$$

Putting (3.9) into (3.6), we have thus shown that

$$
I_{0}(x, N)=\sum_{j=1}^{m}\left\{F_{j, q}(x, N)-F_{j, q}(x, 0)\right\}+A_{q}(x)-K(x, N) .
$$

On the other hand, from (2.3) and an integration by parts, we have 


$$
\begin{aligned}
I_{0}(x, N)= & -\int_{0}^{N} P_{0}^{*}(u) \frac{d}{d u}\left\{(x / u)^{(r+q) / 2} J_{r+q}\left(2\{u x\}^{1 / 2}\right)\right\} d u \\
= & -F_{0, q}(x, N)+F_{0, q}(x, 0)+\int_{0}^{N}(x / u)^{(r+q) / 2} J_{r+q}\left(2\{u x\}^{1 / 2}\right) d P_{0}^{*}(u) \\
(3.12)= & -F_{0, q}(x, N)+F_{0, q}(x, 0)+\sum_{\mu_{n} \leqslant N} b(n)\left(x / \mu_{n}\right)^{(r+q) / 2} J_{r+q}\left(2\left\{\mu_{n} x\right\}^{1 / 2}\right) \\
& -\int_{0}^{N}(x / u)^{(r+q) / 2} J_{r+q}\left(2\{u x\}^{1 / 2}\right)\left\{\frac{1}{2 \pi i} \int_{C^{\prime}} \psi(s) u^{s-1} d s\right\} d u,
\end{aligned}
$$

where $C^{\prime}$ is a cycle encircling the singularities of $\psi(s)$, and since the singularities of $\psi(s)$ lie in $\sigma>0, C^{\prime}$ may be chosen to lie entirely in $\sigma>0$. Combining (3.11) and (3.12), we have

$$
\begin{aligned}
A_{q}(x)+ & \sum_{j=0}^{m}\left\{F_{j, q}(x, N)-F_{j, q}(x, 0)\right\} \\
& +\int_{0}^{N}(x / u)^{(r+q) / 2} J_{r+q}\left(2\{u x\}^{1 / 2}\right)\left\{\frac{1}{2 \pi i} \int_{C^{\prime}} \psi(s) u^{s-1} d s\right\} d u \\
= & \sum_{\mu_{n} \leqslant N} b(n)\left(x / \mu_{n}\right)^{(r+q) / 2} J_{r+q}\left(2\left\{\mu_{n} x\right\}^{1 / 2}\right)+K(x, N) .
\end{aligned}
$$

We now let $N$ tend to $\infty$ in (3.13). We shall examine each expression individually.

Let

$$
I=\int_{0}^{\infty}(x / u)^{(r+q) / 2} J_{r+q}\left(2\{u x\}^{1 / 2}\right)\left\{\frac{1}{2 \pi i} \int_{C^{\prime}} \psi(s) u^{s-1} d s\right\} d u .
$$

Put $v=2(u x)^{1 / 2}$ and formally invert the order of integration to obtain

$$
I=\frac{1}{2 \pi i} \int_{C^{\prime}} \psi(s) x^{r+q-s} 2^{r+q+1-2 s} d s \int_{0}^{\infty} v^{2 s-r-q-1} J_{r+q}(v) d v .
$$

By (2.4), the inner integral converges "at. $\infty$ " provided that $q>2 \sigma-r-3 / 2$. Now, by hypothesis, $q>2 \sigma_{a}^{*}-r-3 / 2$. Since the singularities of $\psi(s)$ have real parts no greater than $\sigma_{a}^{*}, C^{\prime}$ may be chosen so that, indeed, $q>2 \sigma-r$ $-3 / 2$ for all $s \in C^{\prime}$. The inner integral converges "at 0 ," because $\sigma>0$ for all $s \in C^{\prime}$. By a classical theorem [18, p. 349], the inversion in order of integration is justified.

We now evaluate the inner integral of (3.14) by the use of (2.5). By the discussion immediately above, the requirements on $\sigma$ and $\nu=r+q$ are fulfilled, and so we obtain

$$
I=\frac{1}{2 \pi i} \int_{C^{\prime}} \frac{\Gamma(s) \psi(s)}{\Gamma(r+q+1-s)} x^{r+q-s} d s
$$


Next, we calculate $F_{j, q}(x, 0)$ for $0 \leqslant j \leqslant m$. From (3.1) and (2.2), we have

$$
\begin{aligned}
\lim _{y \rightarrow 0} F_{j, q}(x, y) & =-\lim _{y \rightarrow 0} Q_{j}^{*}(y)(x / y)^{(r+q+j) / 2} J_{r+q+j}\left(2\{x y\}^{1 / 2}\right) \\
& =\frac{x^{r+q+j}}{\Gamma(r+q+j+1)} \lim _{y \rightarrow 0} \frac{1}{2 \pi i} \int_{C^{*}} \frac{\Gamma(s) \psi(s)}{\Gamma(s+j+1)} y^{s+j} d s
\end{aligned}
$$

$$
\begin{aligned}
& =-\frac{x^{r+q+j}}{\Gamma(r+q+j+1)} \lim _{y \rightarrow 0} \sum_{\mu=0}^{j} \frac{(-1)^{\mu} \psi(-\mu) y^{j-\mu}}{\mu ! \Gamma(-\mu+j+1)} \\
& =-\frac{x^{r+q+j}(-1)^{j} \psi(-j)}{\Gamma(r+q+j+1) j !}
\end{aligned}
$$

since the singularities of $\psi(s)$ lie in $\sigma>0$. Let $C^{\prime \prime}$ be the image of $C$ under the transformation $s=r-z$. Then, from (3.15), (3.16), (A3), and (2.1),

$$
\begin{aligned}
I-\sum_{j=0}^{m} F_{j, q}(x, 0)= & \frac{1}{2 \pi i} \int_{C^{\prime}} \frac{\Gamma(s) \psi(s)}{\Gamma(r+q+1-s)} x^{r+q-s} d s \\
& +\sum_{j=0}^{m} \frac{(-1)^{j} \psi(-j)}{\Gamma(r+q+j+1) j !} x^{r+q+j} \\
= & \frac{1}{2 \pi i} \int_{C^{\prime \prime}} \frac{\Gamma(s) \psi(s)}{\Gamma(r+q+1-s)} x^{r+q-s} d s \\
& =-\frac{1}{2 \pi i} \int_{C} \frac{\Gamma(z) \phi(z)}{\Gamma(z+q+1)} x^{z+q} d z \\
& =-Q_{q}(x) .
\end{aligned}
$$

For $j>2 \sigma_{a}-r-1 / 2$, we deduce from (3.5) and (2.4) that

$$
\begin{aligned}
F_{j, q}(x, N) & =O\left(N^{(r+j) / 2-1 / 4-(r+q+j) / 2-1 / 4}\right) \\
& =O\left(N^{-(q+1) / 2}\right)=o(1),
\end{aligned}
$$

since $q>-1$, uniformly on any interval $0<x_{1} \leqslant x \leqslant x_{2}<\infty$, as $N$ tends to $\infty$.

Letting $N$ tend to $\infty$ in (3.13) and using (3.2), (3.17), and (3.18), we have so far shown that

$$
P_{q}(x)=\sum_{n=1}^{\infty} b(n)\left(x / \mu_{n}\right)^{(r+q) / 2} J_{r+q}\left(2\left\{\mu_{n} x\right\}^{1 / 2}\right)-\lim _{n \rightarrow \infty} K(x, N) .
$$

Lastly, we must show that $K(x, N)$ tends to 0 as $N$ tends to $\infty$. We must also prove the convergence properties claimed for the infinite series on the right side of (3.3). So far, all expressions which we have shown tend to 0 as 
$N$ tends to $\infty$ tend to 0 uniformly on $\left[x_{1}, x_{2}\right]$.

From (2.4), we have

$$
J_{r+m}\left(\left\{\lambda_{n} / x\right\}^{1 / 2} v\right) J_{r+q+m+1}(v)
$$

$$
\begin{aligned}
= & \frac{2}{\pi v}\left(\frac{x}{\lambda_{n}}\right)^{1 / 4} \cos \left(\left\{\lambda_{n} / x\right\}^{1 / 2} v+c_{1}\right) \cos \left(v+c_{2}\right) \\
& +O\left(\left(x / \lambda_{n}\right)^{1 / 4} v^{-2}\right)+O\left(\left(x / \lambda_{n}\right)^{3 / 4} v^{-2}\right)+O\left(\left(x / \lambda_{n}\right)^{3 / 4} v^{-3}\right) \\
= & \frac{2}{\pi v}\left(\frac{x}{\lambda_{n}}\right)^{1 / 4} \cos \left(\left\{\lambda_{n} / x\right\}^{1 / 2} v+c_{1}\right) \cos \left(v+c_{2}\right)+O\left(\lambda_{n}^{-1 / 4} v^{-2}\right),
\end{aligned}
$$

uniformly for $0<x_{1} \leqslant x \leqslant x_{2}<\infty$, where $c_{1}=-\pi(r+m) / 2-\pi / 4$ and $c_{2}=-\pi(r+q+m+1) / 2-\pi / 4$. The contribution of the $O$-term on the far right side of (3.19) to $K(x, N)$ given by (3.10) is

$$
O\left(\sum_{n=1}^{\infty}|a(n)| \lambda_{n}^{-(r+m) / 2-1 / 4} \int_{2(N x)^{1 / 2}}^{\infty} v^{-q-2} d v\right)=O\left(N^{-(q+1) / 2}\right)=o(1) \text {, }
$$

by (A1) and the fact that $q>-1$, uniformly on $0<x_{1} \leqslant x \leqslant x_{2}<\infty$. The contribution of the main term in (3.19) to $K(x, N)$ is $S_{1}+S_{2}$, where for $j=1,2$,

$$
\begin{aligned}
S_{j}= & \frac{(2 x)^{q}}{\pi} \sum_{n=1}^{\infty} a(n)\left(x / \lambda_{n}\right)^{(r+m) / 2+1 / 4} \\
& \times \int_{2(N x)^{1 / 2}}^{\infty} v^{-q-1} \cos \left\{\left(1+(-1)^{j+1}\left\{\lambda_{n} / x\right\}^{1 / 2}\right) v+c_{2+j}\right\} d v,
\end{aligned}
$$

where $c_{3}=c_{2}+c_{1}$ and $c_{4}=c_{2}-c_{1}$.

We first examine $S_{1}$. Integrating by parts, we have

$$
\begin{aligned}
& \int_{2(N x)}^{\infty} \\
& (3.20)
\end{aligned}
$$

$$
\begin{aligned}
& =\left.\frac{v^{-q-1} \sin \left\{\left(1+\left\{\lambda_{n} / x\right\}^{1 / 2}\right) v+c_{3}\right\}}{1+\left\{\lambda_{n} / x\right\}^{1 / 2}}\right|_{2(N x)^{1 / 2}} ^{\infty}
\end{aligned}
$$

$$
\begin{aligned}
& +\frac{(q+1)}{1+\left\{\lambda_{n} / x\right\}^{1 / 2}} \int_{2(N x)^{1 / 2}}^{\infty} v^{-q-2} \sin \left\{\left(1+\left\{\lambda_{n} / x\right\}^{1 / 2}\right) v+c_{3}\right\} d v \\
& =O\left(N^{-(q+1) / 2}\right)+O\left(N^{-(q+1) / 2}\right)=O\left(N^{-(q+1) / 2}\right),
\end{aligned}
$$

uniformly on $0<x_{1} \leqslant x \leqslant x_{2}<\infty$. Using (A1), we conclude that $S_{1}=$ $O\left(N^{-(q+1) / 2}\right)=o(1)$, uniformly on $\left[x_{1}, x_{2}\right]$.

Now examine $S_{2}$. First, if $q>0$, a trivial estimate yields 


$$
\begin{aligned}
\left|S_{2}\right| & \leqslant \frac{(2 x)^{q}}{\pi} \sum_{n=1}^{\infty}|a(n)|\left(x / \lambda_{n}\right)^{(r+m) / 2+1 / 4} \int_{2(N x)^{1 / 2}}^{\infty} v^{-q-1} d v \\
& =O\left(N^{-q / 2}\right)=o(1),
\end{aligned}
$$

by (A1), uniformly for $0<x_{1} \leqslant x \leqslant x_{2}<\infty$. Now assume that $q \leqslant 0$. If no member of $\left\{\lambda_{n}\right\}, 1 \leqslant n<\infty$, is contained in $\left[x_{1}, x_{2}\right]$, then an integration by parts along the same lines as that in (3.20) readily gives $S_{2}=O\left(N^{-(q+1) / 2}\right)$ $=o(1)$, uniformly on $\left[x_{1}, x_{2}\right]$.

Lastly, we must examine $S_{2}$ in the case when $q=0$ and $\left[x_{1}, x_{2}\right]$ contains at least one member of $\left\{\lambda_{n}\right\}$. In this instance, note that $c_{4}=-\pi / 2$. If $\lambda_{n}=x$, observe that the corresponding integrand in $S_{2}$ is zero. If $\lambda_{n} \neq x$, set $u=\left|1-\left\{\lambda_{n} / x\right\}^{1 / 2}\right| v$ and obtain

$$
\begin{aligned}
\int_{2(N x)^{1 / 2}}^{\infty} v^{-1} \cos \left\{\left(1-\left\{\lambda_{n} / x\right\}^{1 / 2}\right) v-\pi / 2\right\} d v & \\
= & \operatorname{sgn}\left(1-\left\{\lambda_{n} / x\right\}^{1 / 2}\right) \int_{2\left|1-\left\{\lambda_{n} / x\right\}^{1 / 2}\right|(N x)^{1 / 2}}^{\infty} \frac{\sin u}{u} d u .
\end{aligned}
$$

In either case, the integrals in $S_{2}$ are uniformly bounded on $\left[x_{1}, x_{2}\right]$ and tend to 0 as $N$ tends to $\infty$. By (A1), $S_{2}$ converges absolutely and uniformly on $\left[x_{1}, x_{2}\right]$. Hence, in conclusion, $S_{2}$ tends to 0 boundedly on $\left[x_{1}, x_{2}\right]$ as $N$ tends to $\infty$. This completes the proof.

The hypothesis in Theorem 1 on the singularities of $\psi$ is for technical reasons. We can alter the method of proof to dispose of this hypothesis. However, any alteration will impose an upper bound for $m$. Note that assumptions (A1)-(A3) are lower bounds on $m$. For the next theorem, then, we must be able to choose a positive integer $m$ satisfying (A1)-(A3) and

(A4) $m<r+q+1 / 2$.

Put $C^{*}=C_{L} \cup C_{R}$, where $C_{L}$ is a cycle encircling the singularities of $\Gamma(s) \psi(s)$ in the half-plane $\sigma \leqslant 0$, and $C_{R}$ is a cycle in the half-plane $\sigma>0$ encircling the singularities of $\psi(s)$ in $\sigma>0$. Let

$$
T_{\rho}^{*}(x)=A_{\rho}^{*}(x)-\frac{1}{2 \pi i} \int_{C_{R}} \frac{\Gamma(s) \psi(s)}{\Gamma(s+\rho+1)} x^{s+\rho} d x
$$

For $x, y>0, \rho$ real, and an arbitrary nonnegative integer $\nu$, put

$$
G_{\nu, \rho}(x, y)=T_{\nu}^{*}(y)(x / y)^{(r+\rho+\nu) / 2} J_{r+\rho+\nu}\left(2\{x y\}^{1 / 2}\right) .
$$

Since $C_{R} \subset\{s: \sigma>0\}$, we see that $T_{\nu}^{*}(0)=0$ for $\nu \geqslant 0$. Thus, from (2.2), for $\nu \geqslant 0$,

$$
\lim _{y \rightarrow 0} G_{\nu, \rho}(x, y)=0
$$


THEOREM 2. Let $\phi$ and $\psi$ satisfy Definition 1. Suppose that a positive integer $m$ can be chosen to satisfy (A1)-(A4). Suppose that for $2 \sigma_{a}^{*}-r-$ $3 / 2<q \leqslant 2 \sigma_{a}^{*}-r-1 / 2$,

$$
\sum_{0<j<2 \sigma_{a}-r-1 / 2} G_{j, q}(x, N) \rightarrow 0,
$$

uniformly on any finite interval $0<x_{1} \leqslant x \leqslant x_{2}$ as $N$ tends to $\infty$. Then for $\rho>2 \sigma_{a}^{*}-r-3 / 2,(3.3)$ is valid as well as the statements immediately following (3.3).

Proof. Proceed in exactly the same fashion as in the proof of Theorem 1 with $P_{j}^{*}(u)$ replaced by $T_{j}^{*}(u)$ and $F_{j, q}(x, y)$ replaced by $G_{j, q}(x, y)$. When we reach the equation corresponding to (3.8), however, we will have on the right side the additional expression

$$
\begin{aligned}
L(x, N) \equiv \int_{0}^{N}(x / u)^{(r+q+m+1) / 2} J_{r+q+m+1}\left(2\{u x\}^{1 / 2}\right) \\
\times\left\{\frac{1}{2 \pi i} \int_{C_{L}} \frac{\Gamma(s) \psi(s)}{\Gamma(s+m+1)} u^{s+m} d s\right\} d u .
\end{aligned}
$$

Thus, corresponding to (3.13), we have, with the use of (3.21),

$$
\begin{aligned}
A_{q}(x) & +\sum_{j=0}^{m} G_{j, q}(x, N)+L(x, N) \\
& +\int_{0}^{N}(x / u)^{(r+q) / 2} J_{r+q}\left(2\{u x\}^{1 / 2}\right)\left\{\frac{1}{2 \pi i} \int_{C_{R}} \psi(s) u^{s-1} d s\right\} d u \\
& =\sum_{\mu_{n}<N} b(n)\left(x / \mu_{n}\right)^{(r+q) / 2} J_{r+q}\left(2\left\{\mu_{n} x\right\}^{1 / 2}\right)+K(x, N) .
\end{aligned}
$$

As before, we now let $N$ tend to $\infty$. The analysis is the same, except that in lieu of calculating $F_{j, q}(x, 0)$, we must examine

$$
\begin{aligned}
L \equiv \int_{0}^{\infty}(x / u)^{(r+q+m+1) / 2} J_{r+q+m+1}\left(2\{u x\}^{1 / 2}\right) \\
\times\left\{\frac{1}{2 \pi i} \int_{C_{L}} \frac{\Gamma(s) \psi(s)}{\Gamma(s+m+1)} u^{s+m} d s\right\} d u .
\end{aligned}
$$

Put $v=2(u x)^{1 / 2}$ and formally invert the order of integration to obtain

$$
\begin{aligned}
L= & \frac{1}{2 \pi i} \int_{C_{L}} \frac{\Gamma(s) \psi(s)}{\Gamma(s+m+1)} x^{r+q-s} 2^{r+q-m-2 s} d s \\
& \times \int_{0}^{\infty} v^{-r-q+m+2 s J_{r+q+m+1}}(v) d v .
\end{aligned}
$$

The inner integral converges provided that $2 m+2 \sigma+2>0$ and $-r-q+$ $m+2 \sigma-1 / 2<0$. By (A3), we may choose $C_{L}$ so that the former condition 
holds. By (A4), we may choose $C_{L}$ so that the latter condition is fulfilled. The inversion in order of integration is justified, as before, by a theorem in [18, p. 349]. We now evaluate the inner integral in (3.22) by (2.5). Accordingly, we find that

$$
L=\frac{1}{2 \pi i} \int_{C_{L}} \frac{\Gamma(s) \psi(s)}{\Gamma(r+q+1-s)} x^{r+q-s} d s
$$

Thus, corresponding to (3.17), we now have

$$
\begin{array}{r}
\frac{1}{2 \pi i} \int_{C_{R}} \frac{\Gamma(s) \psi(s)}{\Gamma(r+q+1-s)} x^{r+q-s} d s+\frac{1}{2 \pi i} \int_{C_{L}} \frac{\Gamma(s) \psi(s)}{\Gamma(r+q+1-s)} x^{r+q-s} d s \\
=\frac{1}{2 \pi i} \int_{C^{\prime \prime}} \frac{\Gamma(s) \psi(s)}{\Gamma(r+q+1-s)} x^{r+q-s} d s=-Q_{q}(x) .
\end{array}
$$

The remainder of the proof is exactly as before, and so the proof of Theorem 2 is finished.

4. Examples. We give just three short examples by way of illustration of Theorem 1.

EXAMPLE 1. Let

$$
f(\tau)=\sum_{n=1}^{\infty} a(n) e^{2 \pi i n \tau / \lambda} \quad(\operatorname{Im} \tau>0)
$$

be a modular form of dimension $-\kappa, \kappa>0$, and Stufe $\lambda, \lambda>0$, which vanishes at all rational cusps of a fundamental region. R. A. Rankin [24] has shown that there exists a positive number $\alpha$ such that

$$
\sum_{n<x}|a(n)|^{2}=\alpha x^{\kappa}+O\left(x^{\kappa-2 / 5}\right)
$$

as $x$ tends to $\infty$. By the Cauchy-Schwarz inequality, it follows that if

$$
\phi(s)=(2 \pi / \lambda)^{-s} \sum_{n=1}^{\infty} a(n) n^{-s},
$$

then $\sigma_{a} \leqslant(\kappa+1) / 2$. Furthermore, $\phi$ can be analytically continued to an entire function which satisfies the functional equation (2.1) with $\psi=\phi$ and $r=\kappa$. Now, $2 \sigma_{a}-r-3 / 2 \leqslant 2(\kappa+1) / 2-k-3 / 2=-1 / 2$. By (3.2) and (3.4), we then need to show that

$$
P_{0}^{*}(N)=A_{0}(N)=o\left(N^{(k+q) / 2+1 / 4}\right)
$$

for $q>-1 / 2$. Rankin [24] has shown that

$$
A_{0}(N)=O\left(N^{\kappa / 2-1 / 5}\right),
$$

which certainly implies (4.1). (In fact, an earlier, easier result of Hecke's [17], 


$$
A_{0}(N)=O\left(N^{\kappa / 2} \log N\right),
$$

suffices to show (4.1).) Replacing $x$ by $2 \pi x / \lambda$ in (3.3), we deduce that for $\rho>-1 / 2$,

$$
\frac{1}{\Gamma(\rho+1)} \sum_{n \leqslant x}^{\prime} a(n)(x-n)^{\rho}=(\lambda / 2 \pi)^{\rho} \sum_{n=1}^{\infty} a(n)(x / n)^{(\kappa+\rho) / 2} J_{\kappa+\rho}\left(4 \pi\{n x\}^{1 / 2} / \lambda\right) .
$$

EXAMPLE 2. Let $Q(x, y)$ be a positive definite quadratic form with determinant $D$. The Epstein zeta-function $\zeta(s, Q)$ is defined for $\sigma>1$ by

$$
\zeta(s, Q)=\sum_{m, n=-\infty}^{\infty}\{Q(m, n)\}^{-s},
$$

where the prime on the summation sign indicates that the term corresponding to $m=n=0$ is omitted from the summation. P. Epstein [8] has shown that $\zeta(s, Q)$ has an analytic continuation into the entire complex plane that is holomorphic save for a simple pole at $s=1$ with residue $\pi D^{-1 / 2}$. Furthermore, $\zeta(s, Q)$ satisfies the functional equation

$$
\pi^{-s} \Gamma(s) \zeta(s, Q)=D^{-1 / 2} \pi^{s-1} \Gamma(1-s) \zeta\left(1-s, Q^{-1}\right),
$$

where $Q^{-1}$ is the inverse of $Q$. Also, $\zeta(0, Q)=-1$. Let $r(\mu, Q)$ denote the number of distinct pairs $(m, n)$ such that $Q(m, n)=\mu$. Now $2 \sigma_{a}-r-3 / 2$ $=-1 / 2$. Thus, by (3.2) and (3.4), we must show that

$$
P_{0}(N)=o\left(N^{(1+q) / 2+1 / 4}\right)
$$

for $q>-1 / 2$. By a classical theorem of Gauss [10], $P_{0}(N)=O\left(N^{1 / 2}\right)$, which implies (4.2). Hence, replacing $x$ by $\pi x$ in (3.3), we deduce that for $\rho>$ $-1 / 2$,

$$
\begin{aligned}
& \overline{\Gamma(\rho+1)} \sum_{\mu<x}^{\prime} r(\mu, Q)(x-\mu)^{\rho}=\frac{\pi D^{-1 / 2} x^{\rho+1}}{\Gamma(\rho+2)}-\frac{x^{\rho}}{\Gamma(\rho+1)} \\
& +D^{-1 / 2} \pi^{-\rho} \sum_{\mu>0} r\left(\mu, Q^{-1}\right)(x / \mu)^{(\rho+1) / 2} J_{\rho+1}\left(2 \pi\{\mu x\}^{1 / 2}\right)
\end{aligned}
$$

where the values for $\mu$ are arranged in increasing order of magnitude.

For $\rho=0$, (4.3) was first given by Voronoï [25]. Landau ([19], [20], [28, pp. 11-29]) was the first to prove corresponding identities for a much wider class of Epstein zeta-functions associated with positive definite quadratic forms of several variables. See also Hardy's paper ([12], [14, pp. 294-315]) and Walfisz's thesis [26]. Finally, the best possible result, with reference to $\rho$, was established by Chandrasekharan and Narasimhan [5]. This result is also covered by our Theorem 1, but we have chosen to work out the details for only a simple case.

EXAMPLE 3. Let $K=Q(\sqrt{-D})$ be an imaginary quadratic field with 
discriminant $D$. Then the Dedekind zeta-function $\zeta_{K}(s)$ is defined for $\sigma>1$ by $\zeta_{K}(s)=\sum_{n=1}^{\infty} F(n) n^{-s}$, where $F(n)$ is the number of nonzero, integral ideals of norm $n$ in $K . \zeta_{K}(s)$ can be analytically continued into the entire complex plane where $\zeta_{K}(s)$ is holomorphic except for a simple pole of residue $\lambda$ at $s=1$. Furthermore, $\zeta_{K}(s)$ satisfies the functional equation [23, p. 66]

$$
(\sqrt{-D} / 2 \pi)^{s} \Gamma(s) \zeta_{K}(s)=(\sqrt{-D} / 2 \pi)^{1-s} \Gamma(1-s) \zeta_{K}(1-s) .
$$

Again, $2 \sigma_{a}-r-3 / 2=-1 / 2$. Since $P_{0}(N)=O\left(N^{1 / 3}\right)[23, \mathrm{p} .131]$, we see from (3.4) that (3.2) holds. Hence replacing $x$ by $2 \pi x / \sqrt{-D}$ in (3.3), we deduce that for $\rho>-1 / 2$,

$$
\begin{aligned}
\frac{1}{\Gamma(\rho+1)} \sum_{n \leqslant x}^{\prime} F(n)(x-n)^{\rho}=\frac{\lambda x^{\rho+1}}{\Gamma(\rho+2)}+\frac{\zeta_{K}(0) x^{\rho}}{\Gamma(\rho+1)} \\
\quad+(\sqrt{-D} / 2 \pi)^{\rho} \sum_{n=1}^{\infty} F(n)(x / n)^{\rho+1) / 2} J_{\rho+1}\left(4 \pi\{-n x / D\}^{1 / 2}\right) .
\end{aligned}
$$

For $\rho \geqslant 0$, (4.4) was first proven by Walfisz ([26], [27]).

\section{REFERENCES}

1. T. M. Apostol, Identities involving the coefficients of certain Dirichlet series, Duke Math. J. 18 (1951), 517-525. MR 13, 15.

2. Bruce C. Berndt, Identities involving the coefficients of a class of Dirichlet series, Thesis, University of Wisconsin, Madison, Wis., 1966.

3. - Identities involving the coefficients of a class of Dirichlet series. I, Trans. Amer. Math. Soc. 137 (1969), 345-359. MR 38 \#4656.

4. K. Chandrasekharan and S. Minakshisundaram, Typical means, Oxford Univ. Press, London, 1952. MR 14, 1077.

5. K. Chandrasekharan and Raghavan Narasimhan, Hecke's functional equation and arithmetical identities, Ann. of Math. (2) 74 (1961), 1-23. MR 30 \#1988.

6. - Functional equations with multiple gamma factors and the average order of arithmetical functions, Ann. of Math. (2) 76 (1962), 93-136. MR 25 \#3911.

7. A. L. Dixon and W. L. Ferrar, Lattice-point summation formulae, Quart. J. Math. Oxford Ser. 2 (1931), 31-54.

8. Paul Epstein, Zur Theorie allgemeiner Zetafunktionen. II, Math. Ann. 63 (1907), 205-216.

9. A. Erdélyi et al., Tables of integral transforms. Vol. II, McGraw-Hill, New York, 1954. MR 16, 468.

10. C. F. Gauss, De nexu inter multitudinem classium, in quas formae binariae secundi gradus distribuuntur, earumque determinantem, Werke, Zweiter Band, Königlichen Gesellschaft der Wiss. Göttingen, 1876, pp. 269-291.

11. G. H. Hardy, On the expression of a number as the sum of two squares, Quart. J. Math. 46 (1915), 263-283.

12. - The average order of the arithmetical functions $P(x)$ and $\Delta(x)$, Proc. London Math. Soc. (2) 15 (1916), 192-213. 
13. G. H. Hardy, A further note on Ramanujan's arithmetical function $\tau(n)$, Proc. Cambridge Philos. Soc. 34 (1938), 309-315.

14. - Collected papers. Vol. II, Clarendon Press, Oxford, 1967. MR 39 \#3958.

15. G. H. Hardy and E. Landau, The lattice points of a circle, Proc. Roy. Soc. Ser. A 105 (1924), 244-258.

16. E. Hecke, Über die Lösungen der Riemannschen Funktionalgleichung, Math. Z. 16 (1923), 301-307.

17, - Theorie der Eisensteinschen Reihen höherer Stufe und ihre Anwendung auf Funktionentheorie und Arithmetik, Abh. Math. Sem. Univ. Hamburg 5 (1927), 199-224.

18. E. W. Hobson, The theory of functions of a real variable. Vol. II, 2 nd ed., Cambridge Univ. Press, Cambridge, 1926.

19. E. Landau, Über eine Aufgabe aus der theorie der quadratischen Formen, S.-B. Akad. Wiss. Wien Math.-Natur. Kl. 124 (1915), Abt. IIa, 445-468.

20. - Zur analytischen Zahlen theorie der definiten quadratischen Formen. (Über die Gitterpunkte in einem mehrdimensionalen Ellipsoid), S.-B. Königl. Preuss. Akad. Wiss. 31 (1915), 458-476.

21. ᄂ Abhandlung), Nachr. Ges. Wiss. Göttingen Math.-Phys. K1. 1915, 209-243.

22. - Vorlesungen über Zahlentheorie, Zweiter Band, S. Hirzel, Leipzig, 1927.

23. - Einführung in die elementare und analytische Theorie der algebraischen Zahlen und der Ideale, Teubner, Leipzig, 1918.

24. R. A. Rankin, Contributions to the theory of Ramanujan's function $\tau(n)$ and similar arithmetical functions. II. The order of the Fourier coefficients of integral modular forms, Proc. Cambridge Philos. Soc. 35 (1939), 357-372.

25. M. G. Voronoï, Sur la développement, à l'aide des fonctions cylindriques, des sommes doubles $\Sigma f\left(p m^{2}+2 q m n+r n^{2}\right)$, où $p m^{2}+2 q m n+r n^{2}$ est une forme positive à coefficients entiers (Verhandlungen des Dritten Internat. Math.-Kongr. Heidelberg), Teubner, Leipzig, 1905, pp. 241-245.

26. Arnold Walfisz, Über die summatorischen Funktionen einiger Dirichletscher Reihen, Inaugural-Dissertation, Göttingen, 1922.

27. — Über das Piltzsche Teilerproblem in algebraischen Zahlkörpern, Math.

Z. 22 (1925), 153-188.

28. - (editor), Ausgewählte Abhandlungen zur Gitterpunktlehre von Edmund Landau, VEB Deutscher Verlag der Wissenschaften, Berlin, 1962.

29. G. N. Watson, Theory of Bessel functions, 2nd ed., Cambridge Univ. Press, Cambridge; Macmillan, New York, 1944. MR 6, 64.

SCHOOL OF MATHEMATICS, INSTITUTE FOR ADVANCED STUDY, PRINCETON, NEW JERSEY 08540

Current address: Department of Mathematics, University of Illinois, Urbana, Illinois 61801 